\title{
Impulse für die deutsche Pflege von unseren Nachbarn
}

\section{Franz Wagner}

(C) Springer-Verlag Wien 2012

Innerhalb der europäischen Union ist die Ausbildung in der Gesundheits- und Krankenpflege harmonisiert. Das bedeutet, sie wird automatisch in allen EU-Staaten anerkannt. Die Ausbildung in der Gesundheits- und Kinderkrankenpflege sowie Altenpflege werden individuell überprüft und können - falls ein nationales Äquivalent gefunden wird - anerkannt werden.

Dies bedeutet aber noch nicht, dass die Berufsprofile identisch sind. Hier gibt es immer größer werdende Unterschiede. Die Pflegeberufe in Deutschland unterscheiden sich in vielerlei Hinsicht von Pflegeberufen in den anderen EU-Staaten - nicht nur beim Zuschnitt. Die Professionalisierung in den anderen EU-Staaten ist anders verlaufen und hat zumindest im Westen früher begonnen als in Deutsch- land, obwohl nach dem 2. Weltkrieg durchaus ein vergleichbarer Entwicklungsstand vorlag. Derzeit droht Deutschland im internationalen Vergleich eine Sonderentwicklung und damit, abgehängt zu werden.

Europa bedeutet aber nicht nur Wettbewerb um qualifizierte Arbeitskräfte sondern auch ein Markt von Ideen - wir profitieren davon, indem wir von den anderen lernen. Wir können sowohl von den Erfolgen als auch den Fehlern unserer Nachbarn lernen. Durchsetzen müssen wir aber unsere Anliegen selber. Wesentliche Entwicklungstendenzen in anderen EU-Staaten waren die Akademisierung der Ausbildung, Entwicklung von Pflegewissenschaft und -forschung, eine funktionierende (Selbst)-Regulierung und ein klares Rollen- und Aufgabenprofil.

F. Wagner $(\bowtie)$

Bundesgeschäftsführer DBfK e. V.,

Vize-Präsident DPR e. V., Berlin 